
15.06.2020

Keywords

ankle,

rheumatoid arthritis, quality of life,

ultrasound, baropodometry

\section{Submitted: \\ 14.11.2019 \\ Accepted: 16.02.2020 \\ Published: \\ Do ankle, hindfoot, and heel ultrasound findings predict the symptomatology and quality of life in rheumatoid arthritis patients?}

\author{
Oana Șerban¹, Iulia Papp¹, Corina Delia Bocșa1', Mihaela Cosmina Micu², \\ Maria Bădărînză ${ }^{1}$, Adriana Albu' ${ }^{1}$, Daniela Fodor ${ }^{1}$ \\ $12^{\text {nd }}$ Internal Medicine Department, "Iuliu Hatieganu” University of Medicine and Pharmacy, \\ Cluj-Napoca, Romania \\ ${ }^{2}$ Rheumatology Division, Rehabilitation Clinical Hospital, Cluj-Napoca, Romania \\ Correspondence: Daniela Fodor, MD, PhD, 2nd Internal Medicine Department \\ "Iuliu Hatieganu" University of Medicine and Pharmacy, Cluj-Napoca, Romania, \\ 2-4 Clinicilor street, 400006 Cluj-Napoca, Romania; e-mail: dfodor@umfcluj.ro
}

DOI: 10.15557/JoU.2020.0012

\begin{abstract}
Objectives: To evaluate the ankle, hindfoot, and heel changes (determined by physical examination, ultrasound and baropodometry) in patients with rheumatoid arthritis, to compare the findings with healthy subjects, and to analyze if these findings are associated with ankle pain and could affect the quality of life. Methods: We enrolled 35 rheumatoid arthritis patients and 35 healthy controls, and evaluated their ankles (tibiotalar joints, tendons), hindfeet (talonavicular, subtalar joints) and heels using clinical examination, DAS28-CRP, RAPID3 for the evaluation of functional status, quality of life in rheumatoid arthritis questionnaire, ultrasound, and baropodometry. Results: The ultrasound inter-observer agreement was good for the subtalar joint, and very good for the other structures. Flat foot was identified in $50 \%$ of feet in rheumatoid arthritis patients, with $83.8 \%$ having concomitant hindfoot valgus and less subtalar joint synovitis visible from the lateral approach $(32.4 \%$ vs $55.6 \%, p=0.041)$. The body mass index, RAPID3 and subtalar synovitis were independent predictors for the symptomatic ankle (all $p<0.05$ ). Midfoot and heel plantar pressures were higher in rheumatoid arthritis patients compared to healthy controls, but when subtalar synovitis was present, the pressures decreased (avoidance of heel support). Poor quality of life in rheumatoid arthritis patients was independently predicted by DAS28-CRP, RAPID3, disease stage, hindfoot valgus, tibiotalar and subtalar synovitis, tendon pathology, Achilles tendon enthesophytes, calcaneal erosions, plantar fasciitis, and perifasciitis (all $p<0.05$ ). Conclusion: The quality of life of rheumatoid arthritis patients is significantly affected by ankle and hindfoot pathology (inflammatory modifications, but also degenerative findings and deformities). Ultrasound scanning is an important tool in the evaluation of inflammatory and degenerative lesions in these regions, and their early detection might contribute to a better therapeutic management in these patients.
\end{abstract}




\section{Introduction}

Rheumatoid arthritis (RA) is a chronic and progressive systemic disease that leads progressively to joint damage and disability ${ }^{(1,2)}$. Approximately half of patients with RA report foot or ankle joints symptoms as the first manifestation of the disease ${ }^{(3,4)}$, and in time $71 \%$ of these patients develop walking disability ${ }^{(5)}$.

The quality of life (QoL) in RA patients is poorer compared to the general population, and even lower in those with foot involvement, especially regarding mobility, functionality, daily activities, and mental health ${ }^{(3,6)}$. The involvement of the hindfoot (talonavicular [TN] and subtalar [ST] joints) is associated with functional disability and walking difficulties ${ }^{(7)}$. However, feet are seldom examined in RA patients, the main reason being the omission of these joints in the most commonly used disease activity score with 28 joint count (DAS-28), and patients not being routinely referred to a podiatrist ${ }^{(8)}$.

Baropodometry is a method that analyses foot dysfunctions by measuring and mapping the plantar surface pressures during standing and walking ${ }^{(9)}$. RA patients suffer several gait adaptations consisting of reduced walking speed, cadence, stride length and ankle power, double limb support time during walking, and increase of the peak forefoot plantar pressure ${ }^{(10)}$. In order to compensate for their foot dysfunction, they alter the patterns of foot loading by decreasing the duration and velocity of the center of pressure ${ }^{(11)}$. The increase in midfoot plantar pressure is an independent predictor for falls in RA patients ${ }^{(12)}$. There are only a few studies that reported or evaluated plantar pressures in RA patients ${ }^{(13,14)}$.

Ultrasonography (US) is considered to be superior to clinical examination in the detection of synovitis, and its contribution to detecting damage from the early stages, evaluating the therapeutic response, monitoring disease activity or assessing persistent inflammation has been underlined in the European League Against Rheumatism (EULAR) recommendations ${ }^{(15)}$. However, as we previously noted in our systematic review ${ }^{(16)}$, only few studies have been published on US findings for ankle and hindfoot in RA patients. Only one study has explored links between US-detected synovitis (subclinical) of the foot and ankle, and poor QoL and functional status $^{(17)}$.

The aim of the study was to evaluate the ankle, hindfoot, and heel in RA patients using clinical examination, US, and baropodometry, in order to determine the prevalence of lesions, compare the findings with healthy control subjects, and establish which factors have a significant impact on the presence of symptomatic ankle or poor QoL scores. Also, we assessed the role of US in identifying factors that can be influenced to prevent further damage, deformities and foot dysfunction in RA patients.

\section{Material and methods}

\section{Patients and healthy controls}

Consecutive RA patients presenting to the day-hospital department were enrolled between April and December 2018 in this observational, cross-sectional study. The inclusion criteria were: diagnosis of RA according to the 2010 American College of Rheumatology (ACR)/EULAR classification criteria $^{(1)}$, stable treatment in the past 3 months, and age over 18 years. The exclusion criteria included: unstable treatment in the past 3 months before enrollment [need for nonsteroidal anti-inflammatory or antalgic drugs, increase or decrease in oral steroid therapy, synthetic or biological disease-modifying anti-rheumatic drugs (DMARD) initiation, switch or combination, or topical administration of steroid drugs], history of foot and ankle surgery, trauma, tendinosis, other causes of architectural changes within the feet and ankles (e.g. diabetes, osteoarthritis), other inflammatory rheumatoid diseases (e.g. crystal arthropathies, spondylarthritis, etc.), and history of cancer with bone metastasis, including suspect pulmonary nodules where further evaluation was indicated.

The group of patients was compared to healthy control (HC) subjects matched for age, sex, and body mass index (BMI). The HC status was defined as asymptomatic subjects with no history of surgery, trauma, inflammatory and non-inflammatory diseases (including osteoarthritis) on the foot and ankle levels, and no architectural changes of the feet and ankles.

All the patients and subjects provided written informed consent before study enrollment. Also, the approval of the University Ethics Committee, number 51, of 22 January 2018, was obtained.

\section{Clinical examination}

The RA patients were examined by a rheumatologist with 4 years' experience in rheumatology (IP) following a pre-established protocol. Demographic data and the presence of pain at the level of each ankle, quantified by the Visual Analog Scale (VAS), were recorded. The status of symptomatic ankle was established by the presence of pain at the level of the ankle (patient's personal assessment) at the time of enrollment in the study. The swollen and tender joints were counted. The postural deformities of the arches of the feet (flat feet, high arched feet), postural changes of the hindfeet (hindfoot valgus or varus), pain caused by palpation of the anterior, medial, lateral and posterior ankles and hindfeet were assessed. The level of motion, and pain caused by passive or active movements of the ankle (dorsal flexion, plantar flexion, inversion, eversion) and hindfoot joints (inversion, eversion), were also recorded.

\section{Disease activity assessment and blood tests}

The disease activity in RA patients was estimated by calculating the Clinical Disease Activity Index (CDAI) ${ }^{(18)}$ and DAS 28 with CRP (DAS28-CRP) ${ }^{(19)}$. 


\section{Questionnaires}

The functionality and the effect of RA on daily life was assessed by applying the Routine Assessment of Patient Index Data 3 (RAPID3) questionnaire ${ }^{(20)}$, measuring the physical function, the patient's assessment of pain (global, at any site), and overall health. The quality of life was evaluated for each patient using the validated Romanian language version of the RA Quality of Life (RAQoL) questionnaire ${ }^{(21,22)}$ with a score range of 0 to 30 (the higher the score, the lower the QoL).

\section{Baropodometric evaluation}

The plantar pressure measurement was performed by a rheumatologist (CDB) with more than 15 years' experience, using the P-WALK baropodometric platform (BTS S.p.A., Milan, Italy), calibrated according to the manufacturer's recommendations. The plantar pressure distribution and the contact surface of the footprints were measured using static analysis, with the barefoot patient standing still in the middle of the platform for $10 \mathrm{sec}-$ onds, with the feet slightly outlying, forming an angle of about $30^{\circ}$. The Pressure Color Scale for each measurement was preset at $100 \mathrm{kPa}$. The baropodometric evaluation provided an arch index that allowed the classification of each foot in high arch, normal foot or flat foot, a colored map of the foot contact area, and the maximum and average pressure for the $1^{\text {st }}$ toe, $2^{\text {nd }}$ to $5^{\text {th }}$ toes, $1^{\text {st }}$ to $5^{\text {th }}$ metatarsal regions, midfoot (MF), medial heel (MH), and lateral heel (LH). For the purpose of the study, we only used the pressure results obtained at the MF, MH and LH levels.

\section{Ultrasonography}

The US evaluation was performed using GE Logiq S7 ultrasound machine (GE Healthcare, Chicago, USA) with ML6-15 linear transducer (at a frequency adapted to the examined region). In the Power Doppler (PD) mode, the frequency was $9 \mathrm{MHz}$, the gain was set at a minimal level to avoid noise artifacts, and the Pulse Repetition Frequency (PRF) was set at $0.8 \mathrm{kHz}$.

The US examination was performed by two examiners with 5 years' (OS) and 20 years' (DF) of experience in musculoskeletal US, respectively, in a blinded mode for the patient's complaints, or for other results. US images were stored for each structure following a pre-established protocol. In the case of disagreements between the two examiners, a third examiner (MCM), with more than 15 years' experience in musculoskeletal US, reviewed the images.

Following the Outcome Measures in Rheumatology (OMERACT)/EULAR definitions and recommendations ${ }^{(23-28)}$, the tibiotalar (TT), anterior and posterior approaches, talonavicular (TN) and subtalar (ST), medial, lateral and posterior approaches were examined. The synovial hypertrophy ( $\mathrm{SH}$ ) in gray scale, PD signal, osteophytes and erosions were also identified and graded accordingly.

The tibialis tendons, long flexor and extensor tendons and peroneal tendons of the ankles were evaluated following the same guidelines ${ }^{(23)}$. The presence of tenosynovitis, PD signal and tendinosis were also recorded and $\operatorname{graded}^{(29)}$.

In the heel region, the Achilles tendon (enthesophytes, retrocalcaneal bursitis and posterior calcaneal erosions) and plantar fascia (plantar fasciitis, perifasciitis, inferior calcaneal erosions) were evaluated according to the previous recommendations ${ }^{(27,30)}$.

We included the TT joint and tendons in the ankle region, and the TN and ST joints in the hindfoot region. The Achilles tendon, plantar fascia and their adjacent structures were assigned to the heel region.

\section{Statistical analysis}

The sample size was calculated to be 35 for each group at a level of significance of 0.05 and a power of 0.80 . For the estimation of sample size, the rearfoot peak plantar pressure was taken into account ${ }^{(31)}$ with a difference between $\mathrm{RA}$ and $\mathrm{HC}$ groups of $18 \mathrm{kPa}$ and a standard deviation of $30 \mathrm{kPa}$, the effect size of 0.6 , and also the frequencies of ankle synovitis ${ }^{(32)}$ of $18.3 \%$ in the RA group and $2.5 \%$ in the HC group, the effect size of 0.6 .

The assessment for the normal distribution of continuous variables was performed using the ShapiroWilk test. Descriptive statistics were obtained, and the results were presented as numbers (percentages) for the frequencies of the categorical variables, mean \pm standard deviation (SD) for normally distributed continuous variables, and median (interquartile range - IQR) for non-normally distributed continuous variables. Chi-square test or Fisher's exact test (as appropriate) were used to assess the association between categorical variables. The comparison of medians between the two groups was performed using Man-Whitney U test. The inter-observer agreement was assessed using the Cohen's kappa for dichotomous variables and weighted kappa for ordinal variables. The interpretation of the $\mathrm{k}$ coefficient values was as follows: $0-0.20$ poor, 0.20 0.40 fair, $0.40-0.60$ moderate, $0.60-0.80$ good, and $0.80-1$ very good.

Using the univariable and multivariable logistic regression analysis, the unadjusted and adjusted (for the other variables included) odds ratios for the prediction of hindfoot valgus and for the symptomatic ankle were calculated. The predictive variables of RAQoL were established using an ordinal logistic regression model. The variables to be included in the univariable logistic regression analysis were those having clinical relevance. The variables to be incorporated into the multivariable logistic regression models were selected from those that had statistical significance after the univariable 
Tab. 1. Demographic data of rheumatoid arthritis (RA) patients

\begin{tabular}{|c|c|c|c|c|}
\hline & $\begin{array}{c}\text { RA } \\
(n=35 \text { patients })\end{array}$ & $\begin{array}{c}\text { Asymptomatic ankle } \\
\text { ( } n=42 \text { ankles) }\end{array}$ & $\begin{array}{c}\text { Symptomatic ankle } \\
(n=28 \text { ankles) }\end{array}$ & $p$-value* \\
\hline Sex (female) & $30(85.7)$ & $38(90.5)$ & $22(78.6)$ & 0.183 \\
\hline Age & $59.2 \pm 11.25$ & $59.5 \pm 11.48$ & $58.75 \pm 10.88$ & 0.737 \\
\hline BMI & $26.49 \pm 5.64$ & $24.63 \pm 5.18$ & $27.74 \pm 5.59$ & 0.027 \\
\hline Flat foot & $37(52.9)$ & $20(47.6)$ & $17(60.7)$ & 0.282 \\
\hline Calcaneus valgus & 34 (48.6) & $19(45.2)$ & 15 (53.6) & 0.494 \\
\hline Disease duration (mo) & $36(12-132)$ & $42(18.75-105)$ & $36(12-192)$ & 0.981 \\
\hline Seropositive & $31(88.6)$ & $38(90.5)$ & $24(85.7)$ & 0.705 \\
\hline \multicolumn{5}{|l|}{ Disease stage } \\
\hline 1 & $5(14.3)$ & $6(14.3)$ & $4(14.3)$ & \multirow{4}{*}{0.975} \\
\hline II & $11(31.4)$ & $14(33.3)$ & $8(28.6)$ & \\
\hline III & $14(40)$ & $16(38.1)$ & $12(42.9)$ & \\
\hline IV & $5(14.3)$ & $6(14.3)$ & $4(14.3)$ & \\
\hline \multicolumn{5}{|l|}{ Disease duration } \\
\hline$<6$ months & $5(14.3)$ & $4(9.5)$ & $6(21.4)$ & 0.183 \\
\hline 6-12 months & $2(5.7)$ & $4(9.5)$ & 0 & 0.144 \\
\hline $1-2$ years & $4(11.4)$ & $5(11.9)$ & $3(10.7)$ & 0.878 \\
\hline$\geq 2$ years & 24 (68.6) & $29(69.0)$ & $19(67.9)$ & 0.916 \\
\hline Smokers & $6(17.1)$ & $6(14.3)$ & $6(21.4)$ & 0.524 \\
\hline \multicolumn{5}{|l|}{ Treatment } \\
\hline sDMARD & $33(94.3)$ & $40(95.2)$ & $26(92.9)$ & $>0.99$ \\
\hline Cortison & $14(40)$ & $12(28.6)$ & $16(57.1)$ & 0.017 \\
\hline bDMARD & $7(20)$ & $10(23.8)$ & $4(14.3)$ & 0.329 \\
\hline CDAI & $19.87 \pm 12.7$ & $17.05 \pm 11.32$ & $24.11 \pm 13.63$ & 0.059 \\
\hline DAS28-CRP & $3.5 \pm 1.34$ & $3.15 \pm 1.27$ & $4.01 \pm 1.28$ & 0.013 \\
\hline RAPID3 & $11.43 \pm 6.6$ & $9.54 \pm 6.4$ & $14.27 \pm 5.89$ & 0.002 \\
\hline RAQoL & $13(4.5-19.5)$ & $9.5(1-17)$ & 15 (7-22.75) & 0.037 \\
\hline \multicolumn{5}{|c|}{$\begin{array}{l}\text { Data presented as numbers (\%, percentages); mean } \pm \text { standard deviation or median (IQR, interquartile range) as appropriate; BMI - body mass in- } \\
\text { dex; mo - months; sDMARD - synthetic disease modifying drugs; bDMARD - biological disease modifying drugs; VAS - visual analogue scale (0-10); } \\
\text { CDAI - Clinical Disease Activity Index; DAS28-CRP - Disease Activity Score } 28 \text { with C-reactive protein; RAPID3 - Routine Assessment of Patient Index } \\
\text { Data 3; RAQoL - Rheumatoid Arthritis Quality of Life; * Comparison between asymptomatic and symptomatic patients. }\end{array}$} \\
\hline
\end{tabular}

\section{RA patients}

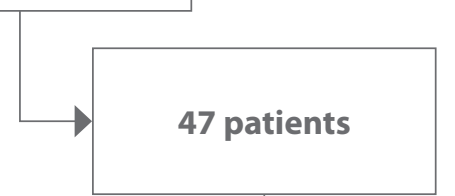

- 8 patients excluded: RA overlap with SpA or SLE and gout

6 patients excluded:

- 1 ankle surgery in the past 8 months

- 1 ankle trauma in the past 5 months

- 3 history of diabetes

- 1 suspect pulmonary nodules

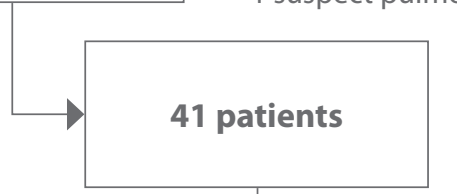

- 4 patients excluded due to idiopathic flat foot before the onset of RA

- 2 patients refused to participate in the study

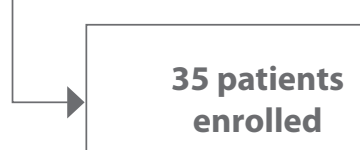

Fig. 1. Patient selection diagram. RA - rheumatoid arthritis; SpA - spondyloarthritis; SLE - systemic lupus erythematosus 
Tab. 2. Comparison of ultrasound findings for the ankle joint, tendon and heel structures between healthy controls (HC) and rheumatoid arthritis (RA) patients

\begin{tabular}{|c|c|c|c|c|c|c|c|}
\hline & US finding & $\begin{array}{c}\mathrm{HC} \\
(n=70)\end{array}$ & $\begin{array}{c}\text { RA } \\
(n=70)\end{array}$ & $p$-value & $\begin{array}{l}\text { Asymptomatic RA } \\
\text { ankle } \\
(n=42 \text { ankles) }\end{array}$ & $\begin{array}{l}\text { Symptomatic RA ankle } \\
\quad(n=28 \text { ankles })\end{array}$ & $p$-value \\
\hline \multicolumn{8}{|l|}{ Joints } \\
\hline \multirow{4}{*}{ TT } & $\mathrm{SH}$ & $8(11.4)$ & $49(70.0)$ & $<0.001$ & $28(66.7)$ & $21(75.0)$ & 0.456 \\
\hline & PD+ & 0 & $8(11.4)$ & 0.006 & $2(4.8)$ & $6(21.4)$ & 0.032 \\
\hline & Osteophytes & $4(5.7)$ & $7(10.0)$ & 0.346 & $5(11.9)$ & $2(7.2)$ & 0.694 \\
\hline & Erosions & 0 & $4(5.7)$ & 0.120 & $3(7.1)$ & $1(3.6)$ & 0.645 \\
\hline \multirow{4}{*}{ ST } & $\mathrm{SH}$ & $3(4.3)$ & $47(67.1)$ & $<0.001$ & 31 (73.8) & $16(57.1)$ & 0.146 \\
\hline & PD+ & 0 & 11 (15.7) & 0.001 & $7(16.7)$ & $4(14.3)$ & 0.789 \\
\hline & Osteophytes & $8(11.4)$ & $1(1.4)$ & 0.033 & 0 & $1(3.6)$ & 0.400 \\
\hline & Erosions & 0 & $5(7.1)$ & 0.058 & $2(4.8)$ & $3(10.7)$ & 0.383 \\
\hline \multirow{4}{*}{ TN } & $\mathrm{SH}$ & $9(12.9)$ & $44(62.9)$ & $<0.001$ & $26(61.9)$ & $18(64.3)$ & 0.840 \\
\hline & PD+ & 0 & 11 (15.7) & 0.001 & $6(14.3)$ & $5(17.9)$ & 0.745 \\
\hline & Osteophytes & $26(37.2)$ & $16(22.9)$ & 0.065 & $10(23.8)$ & $6(21.4)$ & 0.816 \\
\hline & Erosions & 0 & $7(10.0)$ & 0.013 & $4(9.5)$ & $3(10.7)$ & 0.871 \\
\hline \multicolumn{8}{|l|}{ Tendons } \\
\hline \multirow{3}{*}{ TA } & TS & 0 & $5(7.1)$ & 0.048 & $4(9.5)$ & $1(3.6)$ & 0.641 \\
\hline & PD+ & 0 & $1(1.4)$ & 0.316 & 0 & $1(3.6)$ & 0.400 \\
\hline & Tendinosis & $2(2.9)$ & $1(1.4)$ & 0.559 & $1(2.4)$ & 0 & 0.411 \\
\hline \multirow{3}{*}{ EHL } & TS & 0 & $3(4.3)$ & 0.245 & $2(4.8)$ & 1 (3.6) & 0.810 \\
\hline & PD+ & 0 & $2(2.9)$ & 0.496 & $1(2.4)$ & 1 (3.6) & 0.770 \\
\hline & Tendinosis & 0 & 0 & $\mathrm{n} / \mathrm{a}$ & 0 & 0 & $\mathrm{n} / \mathrm{a}$ \\
\hline \multirow{3}{*}{ EDL } & TS & 0 & $2(2.9)$ & 0.496 & $2(4.8)$ & 0 & 0.513 \\
\hline & PD+ & 0 & 0 & $\mathrm{n} / \mathrm{a}$ & 0 & 0 & $\mathrm{n} / \mathrm{a}$ \\
\hline & Tendinosis & 0 & 0 & $\mathrm{n} / \mathrm{a}$ & 0 & 0 & $\mathrm{n} / \mathrm{a}$ \\
\hline \multirow{3}{*}{ TP } & TS & $3(4.3)$ & $13(18.6)$ & 0.008 & $9(21.4)$ & $4(14.3)$ & 0.452 \\
\hline & PD+ & 0 & $3(4.2)$ & 0.245 & $2(4.8)$ & $1(3.6)$ & 0.810 \\
\hline & Tendinosis & $9(12.9)$ & $4(5.7)$ & 0.145 & $2(4.8)$ & $2(7.1)$ & 0.674 \\
\hline \multirow{3}{*}{$\mathrm{FHL}$} & TS & 0 & $3(4.3)$ & 0.245 & $2(4.8)$ & 1 (3.6) & 0.810 \\
\hline & PD+ & 0 & 0 & $\mathrm{n} / \mathrm{a}$ & 0 & 0 & $\mathrm{n} / \mathrm{a}$ \\
\hline & Tendinosis & 0 & 0 & $n / a$ & 0 & 0 & $\mathrm{n} / \mathrm{a}$ \\
\hline \multirow{3}{*}{ FDL } & TS & 0 & $2(2.9)$ & 0.496 & $1(2.4)$ & 1 (3.6) & 0.770 \\
\hline & $\mathrm{PD}+$ & 0 & 0 & $\mathrm{n} / \mathrm{a}$ & 0 & 0 & $\mathrm{n} / \mathrm{a}$ \\
\hline & Tendinosis & 0 & 0 & $\mathrm{n} / \mathrm{a}$ & 0 & 0 & $\mathrm{n} / \mathrm{a}$ \\
\hline \multirow{3}{*}{ Peroneal } & TS & 0 & $7(10.0)$ & 0.013 & $5(11.9)$ & $2(7.1)$ & 0.694 \\
\hline & PD+ & 0 & $1(1.4)$ & 0.316 & $1(2.4)$ & 0 & 0.411 \\
\hline & Tendinosis & 0 & $7(10.0)$ & 0.013 & $4(9.5)$ & $3(10.7)$ & 0.871 \\
\hline \multicolumn{8}{|l|}{ Heel } \\
\hline \multirow{3}{*}{ Achilles tendon } & Enthesophytes & $42(60.0)$ & 55 (78.6) & 0.017 & $33(78.6)$ & $22(78.6)$ & 0.99 \\
\hline & Retrocalcaneal bursitis & 0 & $22(31.4)$ & $<0.001$ & $9(21.4)$ & $13(46.4)$ & 0.027 \\
\hline & $\begin{array}{c}\text { Posterior calcaneal } \\
\text { erosions }\end{array}$ & $4(5.7)$ & $11(15.7)$ & 0.046 & $5(11.9)$ & $6(21.4)$ & 0.328 \\
\hline \multirow{3}{*}{ Plantar fascia } & Plantar fasciitis & $14(20.0)$ & $45(64.3)$ & $<0.001$ & $24(57.1)$ & $21(75)$ & 0.127 \\
\hline & Perifasciitis & 0 & $12(17.1)$ & $<0.001$ & $4(9.5)$ & $8(28.6)$ & 0.038 \\
\hline & $\begin{array}{l}\text { Inferior calcaneal } \\
\text { erosions }\end{array}$ & 0 & $11(15.7)$ & 0.001 & $7(16.7)$ & $4(14.3)$ & 0.789 \\
\hline \multicolumn{8}{|c|}{$\begin{array}{l}\text { Data presented as numbers (\%, percentages); n/a - non-applicable; TT - tibiotalar joint; TN - talonavicular joint; ST - subtalar joint; } \mathrm{TA} \text { - tibialis } \\
\text { anterior; } \mathrm{EHL} \text { - extensor hallucis longus; EDL - extensor digitorum longus; } \mathrm{TP} \text { - tibialis posterior; FHL - flexor hallucis longus; FDL - flexor digitorum } \\
\text { longus; SH - synovial hypertrophy; PD+ - Power Doppler positive; TS - tenosynovitis. }\end{array}$} \\
\hline
\end{tabular}


Tab. 3. Comparison of ultrasound findings for the ankle joint between healthy controls (HC) and rheumatoid arthritis (RA) patients

\begin{tabular}{|c|c|c|c|c|c|c|c|c|}
\hline Joint & $\begin{array}{c}\text { US } \\
\text { finding }\end{array}$ & Grading & $\begin{array}{c}\mathrm{HC} \\
(n=70)\end{array}$ & $\begin{array}{c}\text { RA } \\
(n=70)\end{array}$ & $p$-value & $\begin{array}{c}\text { Asymptomatic RA ankle } \\
(n=42 \text { ankles })\end{array}$ & $\begin{array}{c}\text { Symptomatic } R A \text { ankle } \\
(n=28 \text { ankles })\end{array}$ & $p$-value \\
\hline \multirow{10}{*}{ TT } & \multirow{4}{*}{$\mathrm{SH}$} & Overall & $8(11.4)$ & $49(70.0)$ & $<0.001$ & $28(66.7)$ & $21(75.0)$ & 0.456 \\
\hline & & Grade 1 & $8(11.4)$ & $41(58.6)$ & \multirow{3}{*}{$<0.001$} & $26(61.9)$ & $15(53.6)$ & \multirow{3}{*}{0.097} \\
\hline & & Grade 2 & 0 & $8(11.4)$ & & $2(4.8)$ & $6(21.4)$ & \\
\hline & & Grade 3 & 0 & 0 & & 0 & 0 & \\
\hline & $\mathrm{PD}+$ & Overall & 0 & $8(11.4)$ & 0.006 & $2(4.8)$ & $6(21.4)$ & 0.032 \\
\hline & Osteophytes & Overall & $4(5.7)$ & $7(10.0)^{a}$ & 0.346 & $5(11.9)$ & $2(7.2)$ & 0.694 \\
\hline & \multirow{4}{*}{ Erosions } & Overall & 0 & $4(5.7)$ & 0.120 & $3(7.1)$ & $1(3.6)$ & 0.645 \\
\hline & & Grade 1 & 0 & $3(4.3)$ & \multirow{3}{*}{0.128} & $2(4.8)$ & $1(3.6)$ & \multirow{3}{*}{0.689} \\
\hline & & Grade 2 & 0 & $1(1.4)$ & & $1(2.4)$ & 0 & \\
\hline & & Grade 3 & 0 & 0 & & 0 & 0 & \\
\hline \multirow{10}{*}{ ST } & \multirow{4}{*}{$\mathrm{SH}$} & Overall & $3(4.3)$ & $47(67.1)$ & $<0.001$ & $31(73.8)$ & $16(57.1)$ & 0.146 \\
\hline & & Grade 1 & $3(4.3)$ & $36(51.4)$ & \multirow{3}{*}{$<0.001$} & $24(57.1)$ & $12(42.9)$ & \multirow{3}{*}{0.261} \\
\hline & & Grade 2 & 0 & $10(14.3)$ & & $7(16.7)$ & $3(10.7)$ & \\
\hline & & Grade 3 & 0 & $1(1.4)$ & & 0 & $1(3.6)$ & \\
\hline & PD+ & Overall & 0 & $11(15.7)$ & 0.001 & $7(16.7)$ & $4(14.3)$ & 0.789 \\
\hline & Osteophytes & Overall & $8(11.4)$ & $1(1.4)^{\mathrm{a}}$ & 0.033 & 0 & $1(3.6)$ & 0.400 \\
\hline & \multirow{4}{*}{ Erosions } & Overall & 0 & $5(7.1)$ & 0.058 & $2(4.8)$ & $3(10.7)$ & 0.383 \\
\hline & & Grade 1 & 0 & $2(2.9)$ & \multirow{3}{*}{0.075} & $1(2.4)$ & $1(3.6)$ & \multirow{3}{*}{0.596} \\
\hline & & Grade 2 & 0 & $3(4.3)$ & & $1(2.4)$ & $2(7.2)$ & \\
\hline & & Grade 3 & 0 & 0 & & 0 & 0 & \\
\hline \multirow{10}{*}{ TN } & \multirow{4}{*}{$\mathrm{SH}$} & Overall & $19(12.9)$ & $44(62.9)$ & $<0.001$ & $26(61.9)$ & $18(64.3)$ & 0.840 \\
\hline & & Grade 1 & $9(12.9)$ & $33(47.1)$ & \multirow{3}{*}{$<0.001$} & $20(47.6)$ & $13(46.4)$ & \multirow{3}{*}{0.920} \\
\hline & & Grade 2 & 0 & $11(15.7)$ & & $6(14.3)$ & $5(17.9)$ & \\
\hline & & Grade 3 & 0 & 0 & & 0 & 0 & \\
\hline & PD+ & Overall & 0 & $11(15.7)$ & 0.001 & $6(14.3)$ & $5(17.9)$ & 0.745 \\
\hline & \multirow{4}{*}{ Osteophytes } & Overall & $26(37.2)$ & $16(22.9)$ & 0.065 & $10(23.8)$ & $6(21.4)$ & 0.816 \\
\hline & & Grade 1 & $24(34.3)$ & $15(21.4)$ & \multirow{3}{*}{0.180} & $9(21.4)$ & $6(21.4)$ & \multirow{3}{*}{0.712} \\
\hline & & Grade 2 & $2(2.9)$ & $1(1.4)$ & & $1(2.4)$ & 0 & \\
\hline & & Grade 3 & 0 & 0 & & 0 & 0 & \\
\hline & Erosions & Overall & 0 & 7 (10.0)a & 0.013 & $4(9.5)$ & $3(10.7)$ & 0.871 \\
\hline
\end{tabular}

logistic regression and using the forward method. $P$-values under 0.05 were considered statistically significant. The analysis was performed using Microsoft Excel in Office 365 (Microsoft, Redmond, Washington, SUA), and SPSS Statistics v.23 (IBM, Armonk, New York, USA).

\section{Results}

\section{Demographics of RA patients and HC subjects}

Fifty-five RA patients were examined clinically, but only 35 RA patients were enrolled (Fig. 1). After matching for sex (85.7\% females), age and BMI, a total of $35 \mathrm{HC}$ individuals were recruited ( $\mathrm{HC}$ vs RA: $58.91 \pm 11.59$ vs $59.2 \pm 11.25$ years old, $p=0.743$, and $25.72 \pm 3.40$ vs $26.49 \pm 5.64$ $\left.\mathrm{kg} / \mathrm{m}^{2}, p=0.244\right)$. The RA patients were split into 2 subgroups depending on the presence/absence of symptoms in the ankle region (Tab. 1).

\section{US inter-observer agreement}

The inter-observer agreement for all the US findings in the joint, tendon and heel structures was very good (kappa: 0.82-0.88), except for the subtalar joint, where the level of agreement was good (kappa: 0.71-0.75).

\section{RA vs HC US findings}

The US findings for the ankle/hindfoot joint, tendon and heel structures are summarized in Tab. 2, Tab. 3, Tab. 4, and Tab. 5.

\section{US vs Clinical Examination in RA patients}

The presence of flat foot (37 ankles) was significantly associated with calcaneus valgus (34 ankles) $(p<0.001), 31$ ankles $(83.8 \%)$ having both modifications, and with tibialis posterior (TP) tendinosis $(p=0.029)$. 
Tab. 4. Comparison of ultrasound findings for the ankle tendons between healthy controls (HC) and rheumatoid arthritis (RA) patients

\begin{tabular}{|c|c|c|c|c|c|c|c|c|}
\hline Tendon & US finding & Grading & $\begin{array}{c}\text { HC } \\
(n=70)\end{array}$ & $\begin{array}{c}\text { RA } \\
(n=70)\end{array}$ & $p$-value & $\begin{array}{c}\text { Asymptomatic } R A \text { ankle } \\
(n=42 \text { ankles })\end{array}$ & $\begin{array}{c}\text { Symptomatic RA ankle } \\
(n=28 \text { ankles })\end{array}$ & $p$-value \\
\hline \multirow{6}{*}{ TA } & \multirow{4}{*}{ TS } & Overall & 0 & $5(7.1)$ & 0.048 & $4(9.5)$ & $1(3.6)$ & 0.641 \\
\hline & & Grade 1 & 0 & $4(5.7)$ & \multirow{3}{*}{0.075} & & & \\
\hline & & Grade 2 & 0 & 0 & & & & \\
\hline & & Grade 3 & 0 & $1(1.4)$ & & & & \\
\hline & PD+ & Overall & 0 & $1(1.4)$ & 0.316 & 0 & $1(3.6)$ & 0.400 \\
\hline & Tendinosis & Overall & $2(2.9)$ & $1(1.4)$ & 0.559 & $1(2.4)$ & 0 & 0.411 \\
\hline \multirow{6}{*}{ EHL } & \multirow{4}{*}{ TS } & Overall & 0 & $3(4.3)$ & 0.245 & $2(4.8)$ & $1(3.6)$ & 0.810 \\
\hline & & Grade 1 & 0 & $1(1.4)$ & \multirow{3}{*}{0.225} & & & \\
\hline & & Grade 2 & 0 & $2(2.9)$ & & & & \\
\hline & & Grade 3 & 0 & 0 & & & & \\
\hline & PD+ & Overall & 0 & $2(2.9)$ & 0.496 & $1(2.4)$ & $1(3.6)$ & 0.770 \\
\hline & Tendinosis & Overall & 0 & 0 & $\mathrm{n} / \mathrm{a}$ & 0 & 0 & $\mathrm{n} / \mathrm{a}$ \\
\hline \multirow{3}{*}{ EDL } & TS & Overall & 0 & $2(2.9)^{\mathrm{a}}$ & 0.496 & $2(4.8)$ & 0 & 0.513 \\
\hline & PD+ & Overall & 0 & 0 & $\mathrm{n} / \mathrm{a}$ & 0 & 0 & $\mathrm{n} / \mathrm{a}$ \\
\hline & Tendinosis & Overall & 0 & 0 & $\mathrm{n} / \mathrm{a}$ & 0 & 0 & $\mathrm{n} / \mathrm{a}$ \\
\hline \multirow{6}{*}{ TP } & \multirow{4}{*}{ TS } & Overall & $3(4.3)$ & $13(18.6)$ & 0.008 & $9(21.4)$ & $4(14.3)$ & 0.452 \\
\hline & & Grade 1 & $3(4.3)$ & $11(15.7)$ & \multirow{3}{*}{0.061} & & & \\
\hline & & Grade 2 & 0 & $1(1.4)$ & & & & \\
\hline & & Grade 3 & 0 & $1(1.4)$ & & & & \\
\hline & PD+ & Overall & 0 & $3(4.2)$ & 0.245 & $2(4.8)$ & $1(3.6)$ & 0.810 \\
\hline & Tendinosis & Overall & $9(12.9)$ & $4(5.7)$ & 0.145 & $2(4.8)$ & $2(7.1)$ & 0.674 \\
\hline \multirow{3}{*}{ FHL } & TS & Overall & 0 & $3(4.3)^{\mathrm{a}}$ & 0.245 & $2(4.8)$ & $1(3.6)$ & 0.810 \\
\hline & PD+ & Overall & 0 & 0 & $\mathrm{n} / \mathrm{a}$ & 0 & 0 & $\mathrm{n} / \mathrm{a}$ \\
\hline & Tendinosis & Overall & 0 & 0 & $n / a$ & 0 & 0 & $n / a$ \\
\hline \multirow{3}{*}{ FDL } & TS & Overall & 0 & $2(2.9)^{\mathrm{a}}$ & 0.496 & $1(2.4)$ & $1(3.6)$ & 0.770 \\
\hline & PD+ & Overall & 0 & 0 & $\mathrm{n} / \mathrm{a}$ & 0 & 0 & $\mathrm{n} / \mathrm{a}$ \\
\hline & Tendinosis & Overall & 0 & 0 & $n / a$ & 0 & 0 & $\mathrm{n} / \mathrm{a}$ \\
\hline \multirow{6}{*}{ Peroneal } & \multirow{4}{*}{ TS } & Overall & 0 & $7(10.0)$ & 0.013 & $5(11.9)$ & $2(7.1)$ & 0.694 \\
\hline & & Grade 1 & 0 & $6(8.6)$ & \multirow{3}{*}{0.025} & & & \\
\hline & & Grade 2 & 0 & $1(1.4)$ & & & & \\
\hline & & Grade 3 & 0 & 0 & & & & \\
\hline & PD+ & Overall & 0 & $1(1.4)$ & 0.316 & $1(2.4)$ & 0 & 0.411 \\
\hline & Tendinosis & Overall & 0 & $7(10.0)$ & 0.013 & $4(9.5)$ & $3(10.7)$ & 0.871 \\
\hline
\end{tabular}

Considering only the ankles with calcaneus valgus, ST joint synovitis was detected in $20(58.8 \%)$ ankles: 8 (40\%) from the medial scan, $3(15 \%)$ from the lateral scan, $6(30 \%)$ from both medial and lateral scans, 1 (5\%) from medial and posterior scans, $1(5 \%)$ from lateral and posterior scans, and $1(5 \%)$ from all three scans. Lateral ST synovitis was detected significantly less frequently in patients with hindfoot valgus $(32.4 \%$ vs $55.6 \%, p=0.041)$ (Fig. 2$)$.

Including the relevant parameters in a logistic regression model for the prediction of hindfoot valgus, we found that age $(\mathrm{OR}=1.1, p=0.006)$, disease duration $(\mathrm{OR}=1.02$, $p=0.001)$, and DAS28-CRP $(\mathrm{OR}=1.46, p=0.035)$ were independent predictors of hindfoot valgus.
The rest of the clinical examination was poorly associated with the US findings. The exceptions included pain in the anterior ankle and TT joint synovitis $(p=0.013)$, pain in the Achillean region and enthesophytes $(p=0.018)$, and pain in the inferior heel and plantar fasciitis $(p=0.008)$ or perifasciitis $(p<0.001)$.

\section{Subtalar joint synovitis}

In 47 hindfeet of the RA patients, ST synovitis was identified from the medial approach in 34 (72.3\%) hindfeet, from the lateral approach in $31(66 \%)$, and from the posterior approach in $5(10.6 \%)$. In $15(31.91 \%)$ hindfeet, ST 
Tab. 5. Comparison of ultrasound findings for heel structures between healthy controls (HC) and rheumatoid arthritis (RA) patients

\begin{tabular}{|c|c|c|c|c|c|c|c|c|}
\hline Heel & US finding & Grading & $\begin{array}{c}\text { HC } \\
(n=70)\end{array}$ & $\begin{array}{c}\text { RA } \\
(n=70)\end{array}$ & $p$-value & $\begin{array}{c}\text { Asymptomatic ankle } \\
\quad(n=42 \text { ankles) }\end{array}$ & $\begin{array}{c}\text { Symptomatic ankle } \\
\text { ( } n=28 \text { ankles) }\end{array}$ & $p$-value \\
\hline \multirow{12}{*}{$\begin{array}{l}\text { Achilles } \\
\text { tendon }\end{array}$} & \multirow{4}{*}{ Enthesophytes } & Overall & $42(60.0)$ & $55(78.6)$ & 0.017 & $33(78.6)$ & $22(78.6)$ & 0.99 \\
\hline & & Grade 1 & $40(57.1)$ & $30(42.9)$ & \multirow{3}{*}{$<0.001$} & & & \\
\hline & & Grade 2 & $2(2.9)$ & $24(34.3)$ & & & & \\
\hline & & Grade 3 & 0 & $1(1.4)$ & & & & \\
\hline & \multirow{4}{*}{$\begin{array}{c}\text { Retrocalcaneal } \\
\text { bursitis }\end{array}$} & Overall & 0 & $22(31.4)$ & $<0.001$ & $9(21.4)$ & $13(46.4)$ & 0.027 \\
\hline & & Grade 1 & 0 & $20(28.6)$ & \multirow{3}{*}{$<0.001$} & & & \\
\hline & & Grade 2 & 0 & $2(2.9)$ & & & & \\
\hline & & Grade 3 & 0 & 0 & & & & \\
\hline & \multirow{4}{*}{$\begin{array}{c}\text { Posterior calcane- } \\
\text { al erosions }\end{array}$} & Overall & $4(5.7)$ & $11(15.7)$ & 0.046 & $5(11.9)$ & $6(21.4)$ & 0.328 \\
\hline & & Grade 1 & $4(5.7)$ & $4(5.7)$ & \multirow{3}{*}{0.025} & & & \\
\hline & & Grade 2 & 0 & $7(10.0)$ & & & & \\
\hline & & Grade 3 & 0 & 0 & & & & \\
\hline \multirow{6}{*}{ Plantar fascia } & Plantar fasciitis & Overall & $14(20.0)$ & $45(64.3)$ & $<0.001$ & $24(57.1)$ & $21(75)$ & 0.127 \\
\hline & Perifasciitis & Overall & 0 & $12(17.1)$ & $<0.001$ & $4(9.5)$ & $8(28.6)$ & 0.038 \\
\hline & \multirow{4}{*}{$\begin{array}{l}\text { Inferior calcaneal } \\
\text { erosions }\end{array}$} & Overall & 0 & $11(15.7)$ & 0.001 & $7(16.7)$ & $4(14.3)$ & 0.789 \\
\hline & & Grade 1 & 0 & $8(11.4)$ & \multirow{3}{*}{0.003} & & & \\
\hline & & Grade 2 & 0 & $3(4.3)$ & & & & \\
\hline & & Grade 3 & 0 & 0 & & & & \\
\hline
\end{tabular}

synovitis was visualized both from the medial and lateral approaches, and in $3(6.38 \%)$ hindfeet from all the three approaches.

\section{Symptomatic vs asymptomatic RA patients}

Out of the 35 RA patients, 14 (40\%) were symptomatic, with median (IQR - interquartile range) VAS of 5 (3-7). The distribution of US findings in the asymptomatic and symptomatic ankle groups is detailed in Tab. 2 Tab. 3, Tab. 4 and Tab. 5.

The results of the unadjusted univariable and adjusted multivariable logistic regression for the prediction of the symptomatic ankle are listed in Tab. 6.

\section{Baropodometry in RA patients and HC subjects}

The maximum and mean plantar pressures and contact surfaces in the HC subjects and RA patients (with or without ST synovitis) are shown in supplementary Tab. 7. Significant differences were found in the medial and lateral plantar pressures in the RA patients (with or without ST joint synovitis) (Tab. 8), but in no relation to the US changes in other joints, tendons or heels.

The maximum and mean midfoot pressures were higher in the symptomatic ankle [72 (32.5-96.5) vs 49 (16-129) $\mathrm{kPa}, p=0.043$ and $25(18.5-39.5)$ vs 18 (9-59), $p=0.040$, respectively], and in hindfoot valgus patients [94.5 (29-162) vs 25 (15-95.5), $p=0.046]$. In flat feet, medians of MF maximum and mean pressures [72 (32.5-96.5) vs $49(16-129) \mathrm{kPa}, p=0.043$, and 40 (16-60.5) vs $10(7-25) \mathrm{kPa}, p=0.002$, respectively], MF contact surface $\left[24.15(9.9-31.55)\right.$ vs $6.4(2.7-22.4) \mathrm{cm}^{2}$, $p=0.022]$, $\mathrm{MH}$ maximum and mean plantar pressures [140 (65.5-216) vs $50(34-144) \mathrm{kPa}, p=0.008$, and 76 $(31-85.5)$ vs $24(16-75) \mathrm{kPa}, p=0.005$, respectively], LH maximum and mean pressures [136 (64-210.5) vs $52(33-136) \mathrm{kPa}, p=0.0010$, and $71(30-82.5)$ vs 25 (15-73) $\mathrm{kPa}, p=0.010$, respectively] and total maximum pressures [162.4 (106.7-267.1) vs 52.6 (35.4197.3), $p=0.006]$ were higher comparing with no-flat foot RA patients.

\section{Quality of life of RA patients}

The RAQoL score of RA patients with symptomatic ankle was significantly higher compared to the asymptomatic ankle group $(p=0.037)$. Including all the relevant variables (clinical, US and baropodometric findings) in the multivariable ordinal logistic regression model for the prediction of the RAQoL score, the DAS28-CRP $(p<0.001)$, RAPID3 score $(p<0.001)$, disease stages $(p=0.016)$, hindfoot valgus $(p=0.010)$, ankle joint synovitis [TT synovitis $(p<0.001)$, ST synovitis $(p<0.001)]$, ankle tendon pathology [TP PD positive tenosynovitis $(p=0.029)$, TP tendinosis $(p=0.005)$, peroneal tendinosis $(p<0.001)]$, Achilles tendon enthesophytes $(p<0.001)$, posterior calcaneal erosions $(p=0.011)$, plantar fasciitis $(p=0.04)$, and perifasciitis ( $p=0.007$ ) were found to be independent predictors. 

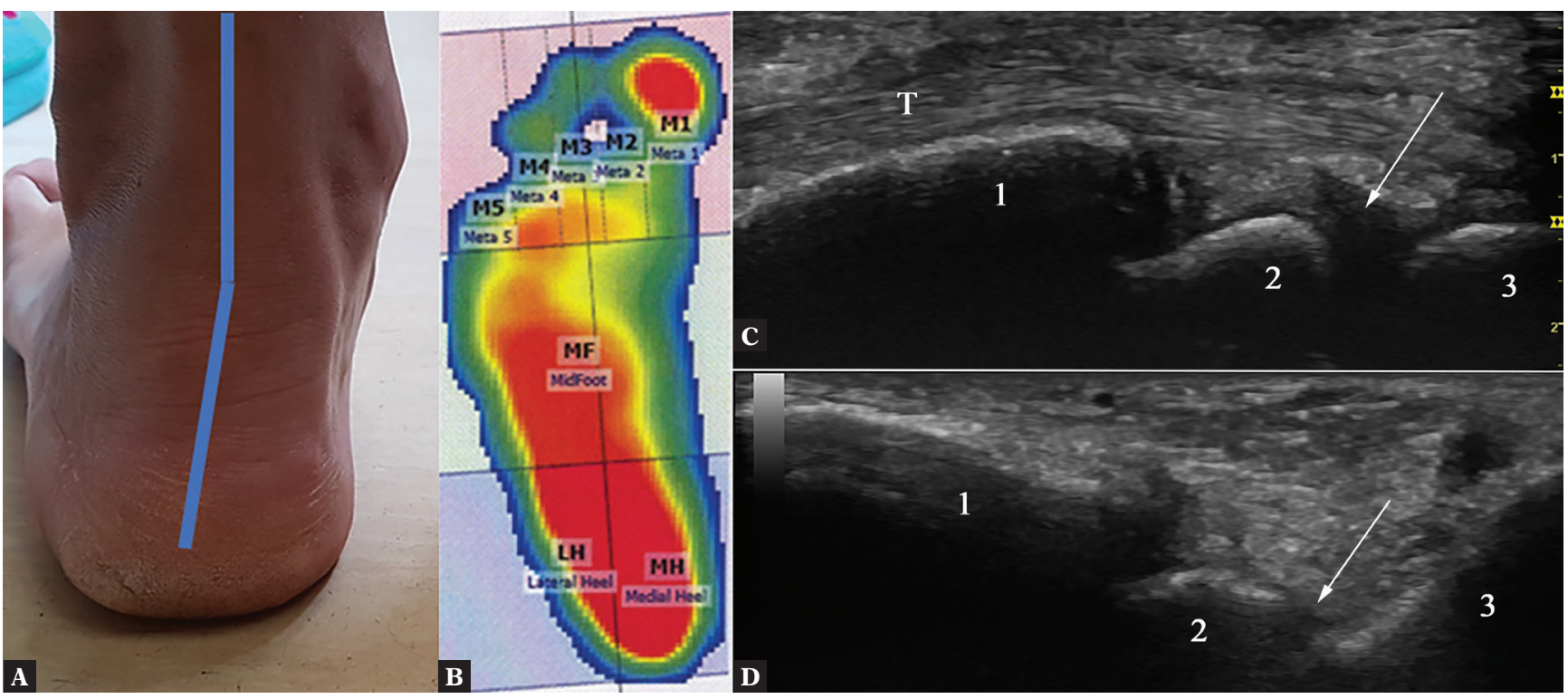

Fig. 2. Example of RA patients with hindfoot valgus. A. Clinical aspect of the left rearfoot of the patient, the blue lines depict the malalignment of the mid-calcaneal axis and mid-tibial axis, with the outward deviation of the mid-calcaneal axis. B. Baropodometric aspect of the left foot showing flat foot pattern with increased surface and pressure of the midfoot (MF) and increased pressure of the lateral and medial heel $(\mathrm{LH}, \mathrm{MH})$ represente by the predominant red color. C. Grade 2 subtalar joint synovitis (arrow) visible from the medial approach; T-extensor hallucis longus tendon; 1 -tibia; 2 -talus; 3 -calcaneus. D. Subtalar joint (arrow) visible from the lateral approach without visible synovitis; 1 - fibula; 2 - talus; 3 - calcaneus

\section{Discussion}

Our study confirmed that the QoL of RA patients is significantly affected by ankle and hindfoot pathology (not only inflammatory, but also degenerative findings and deformities), and that US is an important tool in detecting the majority of these changes. Apart from the DAS-28 calculation system and clinically hindfoot valgus visualization, all the other predictors for poor QoL can be detected/ evaluated by US.

In our study, ST synovitis was a common element found by analyzing the predicting factors for symptomatic ankles, hindfoot deformity, and poor QoL. The data obtained from baropodometry, in relation with ST joints synovitis, offer interesting findings. In patients with ST synovitis, the maximum and mean pressure in the lateral or medial heel were similar to HC, but significantly lower compared to the RA patients without ST synovitis. This observation needs more in-depth exploration.

The mechanism underlying the development of hindfoot valgus in patients with RA is based on ST and TN joint modifications that are stress-related and result from ligament weakening caused by joint inflammation. The main displacements that occur in RA patients' ankles/hindfeet are the plantar flexion of the navicular bone and the lateral, upward shift and valgus rotation of the calcaneus bone ${ }^{(33)}$. Since hindfoot valgus results in a pronated ST joint, meaning

Tab. 6. Logistic regression for the prediction of symptomatic ankle

\begin{tabular}{|c|c|c|c|c|}
\hline Predictor & Unadjusted OR & $p$-value & Adjusted OR* & $p$-value \\
\hline BMI & 1.1 & 0.027 & 1.34 & 0.001 \\
\hline Cortisone therapy & 0.36 & 0.069 & & \\
\hline DAS28-CRP & 1.7 & 0.01 & & \\
\hline RAPID3 & 1.13 & 0.005 & 1.2 & 0.028 \\
\hline Hindfoot valgus & 3.45 & 0.022 & & \\
\hline Flat foot & 1.7 & 0.284 & & \\
\hline TT SH PD+ & 5.45 & 0.048 & & \\
\hline Lat ST SH & 3.03 & 0.033 & 1.93 & 0.007 \\
\hline Post ST SH & 11.18 & 0.03 & & \\
\hline Retrocalcaneal bursitis & 3.18 & 0.03 & & \\
\hline Perifasciitis & 3.8 & 0.047 & & \\
\hline
\end{tabular}


Tab. 7. Differences in the midfoot and rearfoot plantar pressures in rheumatoid arthritis (RA) patients and healthy control (HC) subjects

\begin{tabular}{|c|c|c|c|}
\hline Plantar region & HC & RA & $p$-value \\
\hline \multicolumn{4}{|l|}{ Midfoot } \\
\hline Maximum pressure $(\mathrm{kPa})$ & $34.5(26-48)$ & $84(22-160.5)$ & 0.022 \\
\hline Mean pressure $(\mathrm{kPa})$ & $16(10-21)$ & $27(10-61.5)$ & 0.011 \\
\hline Surface $\left(\mathrm{cm}^{2}\right)$ & $25.55(20.1-33.4)$ & $16.3(5.8-28.9)$ & $<0.001$ \\
\hline \multicolumn{4}{|l|}{ Medial heel } \\
\hline Maximum pressure (kPa) & $71(62-79)$ & $121.5(47-223)$ & 0.115 \\
\hline Mean pressure $(\mathrm{kPa})$ & $37(32-41)$ & $61(21-89)$ & 0.333 \\
\hline Surface $\left(\mathrm{cm}^{2}\right)$ & $20.85(19-22.9)$ & $14.75(10.2-19.2)$ & $<0.001$ \\
\hline \multicolumn{4}{|l|}{ Lateral heel } \\
\hline Maximum pressure $(\mathrm{kPa})$ & $64.5(57-72)$ & $125.5(47-236)$ & 0.052 \\
\hline Mean pressure $(\mathrm{kPa})$ & $34(30-29)$ & $61(23-101)$ & 0.213 \\
\hline Surface $\left(\mathrm{cm}^{2}\right)$ & $21.75(19.8-24.2)$ & $16.35(10.2-19.6)$ & $<0.001$ \\
\hline
\end{tabular}

that the lateral joint space is diminished, either the synovial growth is altered towards the lateral edge of the joint, or the visualization of the synovitis in the lateral scan is impaired by a reduction of the joint space. This might explain why we found less ST synovitis visible from the lateral approach in patients with hindfoot valgus. The presence of synovitis could be an important source of pain ${ }^{(34,35)}$, and probably for this reason the patients had modified walking/standing heel pressure by avoiding heel support (we found that the pressure on the anterior foot in these patients was increased, data not shown). In a 20-year follow-up study, Belt et al. (36,37) $^{(2)}$ radiographically monitored ankle and ST joint damage in patients with early-RA ( $\leq 6$ months), and found that ST joint damage appeared in the first year of follow-up. Most of our patients had stable disease, the majority having over 2 years of disease duration. Probably, an earlier diagnosis of ST synovitis, before the development of permanent deformities, along with effective therapeutic modalities (topical corticosteroid injections, orthosis, rehabilitation therapy, etc.), could have influenced this unfavorable evolution. However, in order to verify this supposition, larger longitudinal studies are needed.

Given its complex anatomy and the lack of experience of the examiners, the US evaluation of the ST joint is quite difficult, and requires good training ${ }^{(28,38)}$. Magnetic resonance imaging (MRI) is a better ankle and hindfoot joint evaluation technique ${ }^{(39,40)}$, but it is expensive, time-consuming and not easily or widely available.

In the clinical practice, the use of US for foot and ankle evaluation in RA is still in its early stages. Recently,

Tab. 8. Medial heel and lateral heel plantar pressure related to the presence of subtalar joint synovitis

\begin{tabular}{|c|c|c|c|}
\hline Plantar region & RA ST- & RA ST+ & $p$-value \\
\hline \multicolumn{4}{|l|}{ Medial heel } \\
\hline Maximum pressure $(\mathrm{kPa})$ & $165(55.5-267.5)$ & $70(41.5-184.5)$ & 0.049 \\
\hline Mean pressure (kPa) & $83(29-134.5)$ & $34(19-81)$ & 0.038 \\
\hline \multicolumn{4}{|l|}{ Lateral heel } \\
\hline Maximum pressure $(\mathrm{kPa})$ & $171(52.5-290)$ & $68(43-180)$ & 0.046 \\
\hline Mean pressure (kPa) & $79(27.5-121)$ & $34(18.5-79.5)$ & 0.048 \\
\hline \multirow[t]{2}{*}{ Total maximum pressure (kPa) } & $203.9(58.85-267.15)$ & $121.1(45.45-193.05)$ & 0.026 \\
\hline & RA lat ST- & RA lat ST+ & \\
\hline \multicolumn{4}{|l|}{ Medial heel } \\
\hline Maximum pressure $(\mathrm{kPa})$ & $127(49.5-237)$ & $35(31-101)$ & 0.034 \\
\hline Mean pressure $(\mathrm{kPa})$ & $70(25-96.5)$ & $16(15-53)$ & 0.048 \\
\hline \multicolumn{4}{|l|}{ Lateral heel } \\
\hline Maximum pressure $(\mathrm{kPa})$ & $131(51-249)$ & $38(31.5-94)$ & 0.030 \\
\hline Mean pressure (kPa) & $67(24.5-106.5)$ & $19(15.5-48.5)$ & 0.048 \\
\hline Total maximum pressure $(\mathrm{kPa})$ & $149.5(54.25-266.25)$ & $36.3(30.25-86.85)$ & 0.008 \\
\hline
\end{tabular}


multidisciplinary recommendations have been published for the diagnosis and treatment of foot conditions in patients with $\mathrm{RA}^{(41)}$ and, despite the lack of evidence (4b level of evidence, based on the "opinion of the expert group") radiological examination is preferred regardless of its known limitations ${ }^{(42)}$. US was considered useful only in cases with inconclusive clinical examination or for injection guiding ${ }^{(41)}$, even though there are studies demonstrating the benefit of US in synovitis (even subclinical) and periarticular structures inflammation detection ${ }^{(39,41,43)}$, and in monitoring the therapeutic response ${ }^{(44)}$.

We often found changes in the heel and surrounding structures, even if the region was not directly damaged in RA. Being superficial, the Achilles tendon and plantar fascia can be easily assessed by US, with very good resolution and reliability ${ }^{(45)}$. Probably mechanical factors are the main cause underlying these changes, but we established that retrocalcaneal bursitis and perifasciitis had a significant effect on the patients' symptomatology and QoL. In the symptomatic patients, US can be used for guiding corticosteroid injection, with no need to change or escalate RA treatment.

It has been shown that pathological findings involving the ankle and hindfoot can be detected in healthy individuals. The hindfoot and ankle, being weight-bearing areas, undergo significant mechanical stress and, for this reason, structural damage might occur. Still, there are limited data regarding the frequency of US modifications in healthy subjects, and available results vary. Luukkainen et al. ${ }^{(46)}$ found TT joint effusion in $4 \%$ of the examined ankles, but no PD signal. Micu et al. (47), in young healthy women, found inflammatory-like US findings in $1.33 \%$ of the examined ST joints and in one TP tendon, but none in the TT and TN joints. Sant'Ana Petterle et al. ${ }^{(43)}$, besides TT and TN joints synovitis (in $1 \%$, and $15 \%$ respectively), found $\mathrm{TN}$ joint erosions (in $2 \%$ of the examined joints). We found synovitis in healthy subjects more frequently $(11.4 \%$ in TT, $4.3 \%$ in $\mathrm{ST}$, and $12.9 \%$ in $\mathrm{TN}$ ), which is probably related to the higher mean age of our healthy group in comparison to the other studies. However, these findings were more common in RA patients, similarly to other published studies $^{(32,43,48)}$.

Inamo et al., ${ }^{(17)}$ retrospectively assessed US-detected subclinical synovitis (overall in feet, ankles and hindfeet joints), and concluded that its presence impaired the functionality and QoL in RA patients. There are no other studies available in the literature regarding the correlation of US findings of the ankles/hindfeet with QoL in RA patients. We established that the ankle and hindfoot synovitis (TT and ST joints), TP tenosynovitis and plantar fasciitis predicted poorer QoL in RA patients. With all being sources for pain, the deformities and dysfunctionality of the feet directly influence the QoL, since the patients' daily activities are impaired.

To the best of our knowledge, our study is the first one to evaluate the relationship between the midfoot and hindfoot plantar pressures and the ankle and hindfoot US findings correlated with the clinical examination.
The study has some limitations. Firstly, the number of patients was small, and the proportion of symptomatic ankles was smaller compared to asymptomatic ankles. We did not use the DAS44 score for the evaluation of our patients (score including ankle and foot evaluation). We chose the DAS28 score following the EULAR/ACR recommendations for reporting disease activity in RA patients ${ }^{(49)}$. The lack of comparison of US findings to other imaging techniques, especially MRI, is another important limitation, but based on very good/good inter-observer agreement - and the involvement of a third experienced examiner for the resolution of disagreements - we may state that the US conclusions are reliable. Since our study was transverse, the relevance of the US findings for patients and the sensitivity to change could not be assessed. We also evaluated the overall functionality of the RA patients, but there are other measuring tools (questionnaires) targeted at the foot functional status. We did not have complete data regarding the health status of the ankles and hindfeet before the onset of RA, which might have biased our results. We did not assess the anterior part of the ST joint as Mandl et al. ${ }^{(28)}$ recommended. We took into consideration the OMERACT recommendations in which the ST joint evaluation should exclude the anterior part of the joint (talocalcaneonavicular joint) ${ }^{(38)}$.

\section{Conclusions}

In RA patients, pathological US findings are more common than in healthy subjects, and the plantar pressures are altered in comparison to healthy individuals, which is probably related to foot deformities or ST joint synovitis. Poor QoL in RA patients is significantly affected by ankle, hindfoot (mainly ST joint) and heel pathologies. US can be used for the detection of inflammatory and degenerative changes in these regions and, together with clinical examination and DAS28CRP calculation, should be considered in all patients.

\section{Acknowledgements}

The authors want to thank Dr. Ioana Felea and Dr. Laura Damian from the Rheumatology Department for their support in the conduct of this study. Also, the authors thank Norina Gâvan from the Podiatry Clinic for providing the baropodometry equipment.

\section{Conflict of interest}

Authors do not report any financial or personal connections with other persons or organizations, which might negatively affect the contents of this publication and/or claim authorship rights to this publication.

\section{Note}

A congress abstract (in Romanian) related to this paper was published in Romania Journal of Rheumatology, vol. XXVIII, Supplement 1, year 2019, page 173 for The XXVI $I^{\text {th }}$ National Congress of Rheumatology in 3-5 October 2019 in Poiana Brasov, Romania. 


\section{References}

1. Aletaha D, Neogi T, Silman AJ, Funovits J, Felson DT, Bingham CO, 3rd et al.: 2010 Rheumatoid arthritis classification criteria: an American College of Rheumatology/European League Against Rheumatism collaborative initiative. Arthritis Rheum 2010; 62: 2569-2581.

2. Smolen JS, Landewé R, Bijlsma J, Burmester G, Chatzidionysiou K, Dougados $\mathrm{M}$ et al.: EULAR recommendations for the management of rheumatoid arthritis with synthetic and biological disease-modifying antirheumatic drugs: 2016 update. Ann Rheum Dis 2017; 76: 960-977.

3. Yano K, Ikari K, Inoue E, Sakuma Y, Mochizuki T, Koenuma N et al.: Features of patients with rheumatoid arthritis whose debut joint is a foot or ankle joint: A 5,479-case study from the IORRA cohort. PLoS One 2018; 13: e0202427.

4. Otter SJ, Lucas K, Springett K, Moore A, Davies K, Cheek L et al: Foot pain in rheumatoid arthritis prevalence, risk factors and management: an epidemiological study. Clin Rheumatol 2010; 29: 255-271.

5. Grondal L, Tengstrand B, Nordmark B, Wretenberg P, Stark A: The foot: still the most important reason for walking incapacity in rheumatoid arthritis: distribution of symptomatic joints in 1,000 RA patients. Acta Orthop 2008; 79: 257-261.

6. Wickman AM, Pinzur MS, Kadanoff R, Juknelis D: Health-related quality of life for patients with rheumatoid arthritis foot involvement. Foot Ankle Int 2004; 25: 19-26.

7. Jeong HJ, Sohn IW, Kim D, Cho SK, Park SB, Sung IH et al.: Impact of midfoot and Hindfoot involvement on functional disability in Korean patients with rheumatoid arthritis. BMC Musculoskelet Disord 2017; 18: 365 .

8. de Souza S, Williams R, Lempp H: Patient and clinician views on the quality of foot health care for rheumatoid arthritis outpatients: a mixed methods service evaluation. J Foot Ankle Res 2016; 9: 1.

9. Rosário JL: A review of the utilization of baropodometry in postural assessment. J Bodyw Mov Ther 2014; 18: 215-219.

10. Carroll M, Parmar P, Dalbeth N, Boocock M, Rome K: Gait characteristics associated with the foot and ankle in inflammatory arthritis: a systematic review and meta-analysis. BMC Musculoskelet Disord 2015; 16: 134.

11. Semple R, Turner DE, Helliwell PS, Woodburn J: Regionalised centre of pressure analysis in patients with rheumatoid arthritis. Clin Biomech (Bristol, Avon) 2007; 22: 127-129.

12. Brenton-Rule A, Dalbeth N, Menz HB, Bassett S, Rome K: Foot and ankle characteristics associated with falls in adults with established rheumatoid arthritis: a cross-sectional study. BMC Musculoskelet Disord 2016; 17: 22 .

13. Turner DE, Woodburn J, Helliwell PS, Cornwall MW, Emery P: Pes planovalgus in RA: a descriptive and analytical study of foot function determined by gait analysis. Musculoskeletal Care 2003; 1: 21-33.

14. Stewart S, Carroll M, Brenton-Rule A, Keys M, Bell L, Dalbeth N et al.: Region-specific foot pain and plantar pressure in people with rheumatoid arthritis: A cross-sectional study. Clin Biomech (Bristol, Avon) 2018; 55: 14-17.

15. Colebatch AN, Edwards CJ, Ostergaard M, van der Heijde D, Balint PV, D'Agostino MA et al.: EULAR recommendations for the use of imaging of the joints in the clinical management of rheumatoid arthritis. Ann Rheum Dis 2013; 72: 804-814.

16. Serban $\mathrm{O}$, Bădărînză M, Fodor D: The relevance of ultrasound examination of the foot and ankle in patients with rheumatoid arthritis - a review of the literature. Med Ultrason 2019; 21: 175-182.

17. Inamo J, Kaneko Y, Sakata K, Takeuchi T: Impact of subclinical synovitis in ankles and feet detected by ultrasonography in patients with rheumatoid arthritis. Int J Rheum Dis 2019; 22: 62-67.

18. Aletaha D, Nell VP, Stamm T, Uffmann M, Pflugbeil S, Machold K et al.: Acute phase reactants add little to composite disease activity indices for rheumatoid arthritis: validation of a clinical activity score. Arthritis Res Ther 2005; 7: R796-806.

19. Fransen J, Welsing P, De Keijzer R, Van Riel P: Disease activity scores using C-reactive protein: CRP may replace ESR in the assessment of RA disease activity [abstract]. Ann Rheum Dis 2004; 62: 151.

20. Pincus T, Swearingen CJ, Bergman M, Yazici Y: RAPID3 (Routine Assessment of Patient Index Data 3), a rheumatoid arthritis index without formal joint counts for routine care: proposed severity categories compared to disease activity score and clinical disease activity index categories. J Rheumatol 2008; 35: 2136-2147.

21. Whalley D, McKenna SP, de Jong Z, van der Heijde D: Quality of life in rheumatoid arthritis. Br J Rheumatol 1997; 36: 884-888.

22. Wilburn J, McKenna SP, Twiss J, Rouse M, Korkosz M, Jancovic R et al.: Further international adaptation and validation of the Rheumatoid Arthritis Quality of Life (RAQoL) questionnaire. Rheumatol Int 2015; 35: 669-675.

23. Wakefield RJ, Balint PV, Szkudlarek M, Filippucci E, Backhaus M, D'Agostino MA et al.: Musculoskeletal ultrasound including definitions for ultrasonographic pathology. J Rheumatol 2005; 32: 2485-2487.

24. Szkudlarek M, Court-Payen M, Jacobsen S, Klarlund M, Thomsen HS, Østergaard M: Interobserver agreement in ultrasonography of the finger and toe joints in rheumatoid arthritis. Arthritis Rheum 2003; 48: 955-962.

25. Mathiessen A, Haugen IK, Slatkowsky-Christensen B, Bøyesen P, Kvien TK, Hammer HB: Ultrasonographic assessment of osteophytes in 127 patients with hand osteoarthritis: exploring reliability and associations with MRI, radiographs and clinical joint findings. Ann Rheum Dis 2013; 72: 51-56.

26. D'Agostino MA, Terslev L, Aegerter P, Backhaus M, Balint P, Bruyn GA et al.: Scoring ultrasound synovitis in rheumatoid arthritis: a EULAROMERACT ultrasound taskforce-Part 1: definition and development of a standardised, consensus-based scoring system. RMD Open 2017; 3: e000428.

27. Möller I, Janta I, Backhaus M, Ohrndorf S, Bong DA, Martinoli C et al:: The 2017 EULAR standardised procedures for ultrasound imaging in rheumatology. Ann Rheum Dis 2017; 76: 1974-1979.

28. Mandl P, Bong D, Balint PV, Hammer HB, Miguel M, Naredo E et al.: Sonographic and anatomic description of the subtalar joint. Ultrasound Med Biol 2018; 44: 119-123.

29. Naredo E, D’Agostino MA, Wakefield RJ, Moller I, Balint PV, Filippucci E et al.: Reliability of a consensus-based ultrasound score for tenosynovitis in rheumatoid arthritis. Ann Rheum Dis 2013; 72: 1328-1334.

30. Balint PV, Terslev L, Aegerter P, Bruyn GAW, Chary-Valckenaere I, Gandjbakhch F et al.: Reliability of a consensus-based ultrasound definition and scoring for enthesitis in spondyloarthritis and psoriatic arthritis: an OMERACT US initiative. Ann Rheum Dis 2018; 77: 1730-1735.

31. Tuna H, Birtane M, Tastekin N, Kokino S: Pedobarography and its relation to radiologic erosion scores in rheumatoid arthritis. Rheumatol Int 2005; 26: 42-47.

32. Elsaman AM, Mostafa ES, Radwan AR: Ankle evaluation in active rheumatoid arthritis by ultrasound: a cross-sectional study. Ultrasound Med Biol 2017; 43: 2806-2813.

33. Liu H, Sugamoto K, Itohara T, Tomita T, Hashimoto J, Yoshikawa H: In vivo three-dimensional skeletal alignment analysis of the hindfoot valgus deformity in patients with rheumatoid arthritis. J Orthop Res 2007; 25: 330-339.

34. Baker JF, Conaghan PG, Emery P, Baker DG, Ostergaard M: Relationship of patient-reported outcomes with MRI measures in rheumatoid arthritis. Ann Rheum Dis 2017; 76: 486-490.

35. Enache L, Popescu CC, Micu M, Cojocaru A, Suta VC, Suta M et al.: Ankle involvement in rheumatoid arthritis - a comparison of inflammatory signs on musculoskeletal ultrasound and magnetic resonance imaging. Med Ultrason 2019; 21: 265-272. doi:10.11152/mu-2038.

36. Belt EA, Kaarela K, Maenpaa H, Kauppi MJ, Lehtinen JT, Lehto MU: Relationship of ankle joint involvement with subtalar destruction in patients with rheumatoid arthritis. A 20-year follow-up study. Joint Bone Spine 2001; 68: 154-157.

37. Belt EA, Kaarela K, Kauppi MJ: A 20-year follow-up study of subtalar changes in rheumatoid arthritis. Scand J Rheumatol 1997; 26: 266-268.

38. Bruyn GAW, Siddle HJ, Hanova P, Costantino F, Iagnocco A, Sedie AD et al.: Ultrasound of subtalar joint synovitis in patients with rheumatoid arthritis: results of an OMERACT reliability exercise using consensual definitions. J Rheumatol 2019; 46: 351-359.

39. Wakefield RJ, Freeston JE, O'Connor P, Reay N, Budgen A, Hensor EM et al.: The optimal assessment of the rheumatoid arthritis hindfoot: a comparative study of clinical examination, ultrasound and high field MRI. Ann Rheum Dis 2008; 67: 1678-1682.

40. Serban O, Fodor D, Papp I, Micu M, Duma D, Csutak C et al.: Reasons for discordances between ultrasonography and magnetic resonance im- 
aging in the evaluation of the ankle, hindfoot and heel of the patients with rheumatoid arthritis. Med Ultrason 2019; 21: 405-413.

41. Tenten-Diepenmaat M, van der Leeden M, Vliet Vlieland TPM, Dekker J, Group RAFE: Multidisciplinary recommendations for diagnosis and treatment of foot problems in people with rheumatoid arthritis. J Foot Ankle Res 2018; 11: 37.

42. Wilkinson VH, Rowbotham EL, Grainger AJ: Imaging in foot and ankle arthritis. Semin Musculoskelet Radiol 2016; 20: 167-174.

43. Sant'Ana Petterle G, Natour J, Rodrigues da Luz K, Soares Machado F, dos Santos MF, da Rocha Correa Fernandes A et al.: Usefulness of US to show subclinical joint abnormalities in asymptomatic feet of RA patients compared to healthy controls. Clin Exp Rheumatol 2013; 31: 904-912.

44. D'Agostino MA, Terslev L, Wakefield R, Ostergaard M, Balint P, Naredo E et al.: Novel algorithms for the pragmatic use of ultrasound in the management of patients with rheumatoid arthritis: from diagnosis to remission. Ann Rheum Dis 2016; 75: 1902-1908.

45. Aguila Maldonado R, Ruta S, Valuntas ML, Garcia M: Ultrasonography assessment of heel entheses in patients with spondyloarthritis: a com- parative study with magnetic resonance imaging and conventional radiography. Clin Rheumatol 2017; 36: 1811-1817.

46. Luukkainen R, Ekman P, Luukkainen P, Koski JM: Ultrasonographic findings in metatarsophalangeal and talocrural joints in healthy persons. Clin Rheumatol 2009; 28: 311-313.

47. Micu MC, Fodor D, Micu R, Bolboacča SD, Ionescu R: Pregnant versus non-pregnant healthy subjects - a prospective longitudinal musculoskeletal ultrasound study concerning the spectrum of normality. Med Ultrason 2018; 20: 319-327.

48. Gutierrez M, Pineda C, Salaffi F, Raffeiner B, Cazenave T, Martinez-Nava GA et al.: Is ankle involvement underestimated in rheumatoid arthritis? Results of a multicenter ultrasound study. Clin Rheumatol 2016; 35: 2669-2678.

49. Aletaha D, Landewe R, Karonitsch T, Bathon J, Boers M, Bombardier C et al.: Reporting disease activity in clinical trials of patients with rheumatoid arthritis: EULAR/ACR collaborative recommendations. Ann Rheum Dis 2008; 67: 1360-1364. 